\title{
Entangled-photon ellipsometry
}

\author{
Ayman F. Abouraddy, Kimani C. Toussaint, Jr., Alexander V. Sergienko, Bahaa E. A. Saleh, and \\ Malvin C. Teich \\ Quantum Imaging Laboratory, Departments of Electrical \& Computer Engineering and Physics, Boston University, \\ Boston, Massachusetts 02215-2421
}

Received May 25, 2001; revised manuscript received October 10, 2001

\begin{abstract}
Performing reliable measurements in optical metrology, such as those needed in ellipsometry, requires a calibrated source and detector, or a well-characterized reference sample. We present a novel interferometric technique to perform reliable ellipsometric measurements. This technique relies on the use of a nonclassical optical source, namely, polarization-entangled twin photons generated by spontaneous parametric downconversion from a nonlinear crystal, in conjunction with a coincidence-detection scheme. Ellipsometric measurements acquired with this scheme are absolute, i.e., they require neither source nor detector calibration, nor do they require a reference. (C) 2002 Optical Society of America

OCIS codes: $120.2130,270.0270,190.0190,120.3940,000.1600,350.4600$.
\end{abstract}

\section{INTRODUCTION}

A question that arises frequently in metrology is the following: How does one measure reliably the reflection or transmission coefficient of an unknown sample? The outcome of such a measurement depends on the reliability of both the source and the detector used to carry out the measurements. If they are each absolutely calibrated, such measurements would be trivial. Since such ideal conditions are never met in practice, and since highprecision measurements are often required, a myriad of experimental techniques, such as null and interferometric approaches, have been developed to circumvent the imperfections of the devices involved in these measurements.

One optical metrology setting in which high-precision measurements are a necessity is ellipsometry, ${ }^{1-6}$ in which the polarization of light is used to study thin films on substrates, a technique established more than a hundred years ago. ${ }^{1,4,5}$ Ellipsometers have proven to be an important metrological tool in many arenas ranging from the semiconductor industry to biomedical applications. To carry out ideal ellipsometry, one needs a perfectly calibrated source and detector. Various approaches, such as null and interferometric techniques, have been commonly used in ellipsometers ${ }^{2,6}$ to approach this ideal. Section 2 of this paper describes the basic requirements for ideal ellipsometry and reviews some of the more common techniques that have been used in conjunction with available detectors and sources.

In this paper we propose a novel technique for obtaining reliable ellipsometric measurements based on the use of twin photons produced by the process of spontaneous optical parametric downconversion ${ }^{7-11}$ (SPDC). This source has been used effectively in studies of the foundations of quantum mechanics ${ }^{12,13}$ and in applications in quantum metrology ${ }^{14-17}$; quantum information processing, such as quantum cryptography ${ }^{18-20}$; quantum teleportation $^{21,22}$; and quantum imaging. ${ }^{23-25}$ We extend the use of this nonclassical light source to the field of ellipsometry.

In Section 3 we propose two different experimental implementations of twin-photon ellipsometry. The first makes use of a twin-photon interferometer that has been previously used for testing the foundations of quantum mechanics. The second technique makes direct use of polarization-entangled photon pairs emitted through SPDC. This approach effectively comprises an interferometric ellipsometer, although none of the optical elements usually associated with constructing an interferometer are utilized. Instead, polarization entanglement itself is harnessed to perform interferometry and to achieve ideal ellipsometry. The inherent limitations of the first technique are eliminated in the second.

\section{IDEAL ELLIPSOMETRY}

In an ideal ellipsometer, the light emitted from a reliable optical source is directed into an unknown optical system (which may simply be an unknown sample that reflects the impinging light) and thence into a reliable detector. The practitioner keeps track of the emitted and detected radiation, and from this bookkeeping (s)he can infer information about the optical system. This device may be used as an ellipsometer if the source can emit light in any specified state of polarization. The sample is characterized by two parameters: $\psi$ and $\Delta$. The quantity $\psi$ is related to the magnitude of the ratio of the sample's eigenpolarization complex reflection coefficients, $\widetilde{r}_{1}$ and $\widetilde{r}_{2}$, through $\tan \psi=\left|\widetilde{r}_{1}\right| \widetilde{r}_{2} \mid ; \Delta$ is the phase shift between them. ${ }^{2}$

Because of the high accuracy required in measuring these parameters, an ideal ellipsometric measurement would require absolute calibration of both the source and the detector. Since this is not attainable in practical settings, ellipsometry makes use of a myriad of experimental techniques developed to circumvent the imperfections of the involved devices. The most common techniques are null and interferometric ellipsometry. 
In the traditional null ellipsometer, ${ }^{2}$ depicted in Fig. 1, the sample is illuminated with a beam of light that can be prepared in any state of polarization. The reflected light, which is generally elliptically polarized, is then analyzed. The polarization of the incident beam is adjusted to compensate for the change in the relative amplitude and phase, introduced by the sample, between the two eigenpolarizations; thus the resulting reflected beam is linearly polarized. If passed through an orthogonal linear polarizer, this linearly polarized beam will yield a null (zero) measurement at the optical detector. The null ellipsometer does not require a calibrated detector since it does not measure intensity but instead records a null. The principal drawback of null measurement techniques is the need for a reference to calibrate the null, for example, to find its initial location (the rotational axis of reference at which an initial null is obtained) and then to compare this with the subsequent location upon inserting the sample into the apparatus. Such a technique thus alleviates the problem of an unreliable source and detector but necessitates the use of a reference sample. The accuracy and reliability of all measurements depend on our knowledge of this reference sample. In this case, the measurements are a function of $\psi, \Delta$, and the parameters of the reference sample.

Another possibility is to perform ellipsometry that employs an interferometric configuration in which the light from the source follows more than one path, usually created by beam splitters, before reaching the detector. The sample is placed in one of those paths. We can then estimate the efficiency of the detector (assuming a reliable source) by performing measurements when the sample is removed from the interferometer. This configuration thus alleviates the problem of an unreliable detector but depends on the reliability of the source and suffers from the drawback of requiring several optical components (beam splitters, mirrors, etc.). The ellipsometric measurements are a function of $\psi, \Delta$, source intensity, and the parameters of the optical elements. The accuracy of the measurements are therefore limited by our knowledge of the parameters characterizing these optical components. The stability of the optical arrangement is also of importance to the performance of such a device.

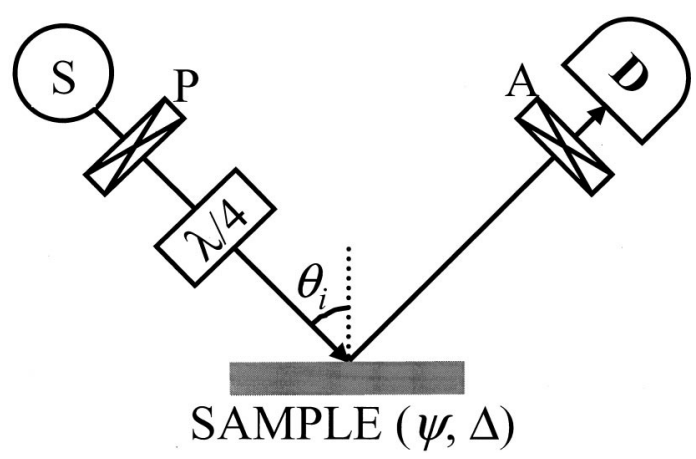

Fig. 1. Null ellipsometer: $\mathrm{S}$ is an optical source, $\mathrm{P}$ is a linear polarizer, $\lambda / 4$ is a quarter-wave plate (compensator), $\mathrm{A}$ is a linear polarization analyzer, and $\mathrm{D}$ is an optical detector; $\theta_{i}$ is the angle of incidence. The sample is characterized by the ellipsometric parameters $\psi$ and $\Delta$ defined in the text.

\section{TWIN-PHOTON ELLIPSOMETRY}

All classical optical sources (including ideal amplitudestabilized lasers) suffer from unavoidable quantum fluctuations even if all other extraneous noise sources are removed. Fluctuations in the photon number can only be eliminated by constructing a source that emits nonoverlapping wave packets, each of which contains a fixed photon number. Such sources have been investigated, and indeed sub-Poisson light sources have been demonstrated. ${ }^{26-28}$

One such source may be readily realized through the process of spontaneous parametric downconversion (SPDC) from a second-order nonlinear crystal (NLC) when illuminated with a monochromatic laser beam (pump). ${ }^{11}$ A portion of the pump photons disintegrate into photon pairs. The two photons that comprise the pair, known as signal and idler, are highly correlated since they conserve the energy (frequency matching) and momentum (phase matching) of the parent pump photon.

In type II SPDC the signal and idler photons have orthogonal polarizations, one extraordinary and the other ordinary. These two photons emerge from the NLC with a relative time delay due to the birefringence of the NLC. ${ }^{29}$ Passing the pair through an appropriate birefringent material of suitable length compensates for this time delay. This temporal compensation is required for extracting $\psi$ and $\Delta$ from the measurements; we show subsequently that when compensation is not employed, one may obtain $\psi$ but not $\Delta$.

The signal and idler may be emitted in two different directions, a case known as noncollinear SPDC, or in the same direction, a case known as collinear SPDC. In the former situation, the SPDC state is polarization entangled; its quantum state is described by $^{29}$

$$
|\Psi\rangle=\frac{1}{\sqrt{2}}(|H V\rangle+|V H\rangle),
$$

where $H$ and $V$ represent horizontal and vertical polarizations, respectively. ${ }^{30}$ It is understood that the first polarization indicated in a ket is that of the signal photon, and the second is that of the idler. Such a state may not be written as the product of states of the signal and idler photons. Although Eq. (1) represents a pure quantum state, the signal and idler photons considered separately are each unpolarized. ${ }^{31,32}$ The state represented in Eq. (1) assumes that there is no relative phase between the two kets. Although the relative phase may not be zero, it can, in general, be arbitrarily chosen by making small adjustments to the NLC.

In the collinear case the SPDC state is in a polarization-product state,

$$
|\Psi\rangle=|H V\rangle .
$$

Because this state is factorizable (i.e., it may be written as the product of states of the signal and idler photons), it is not entangled.

We first discuss a configuration based on the use of collinear type II SPDC, which we call an unentangled twinphoton ellipsometer. This configuration is introduced for pedagogical reasons as a precursor to the configuration of principal interest to us, called the entangled twin-photon 
ellipsometer, which makes use of polarization-entangled photon pairs (noncollinear type II SPDC). Both arrangements are described with a generalization of the Jonesmatrix formalism appropriate for twin-photon polarized beams.

\section{A. Unentangled Twin-Photon Ellipsometer}

We now examine the use of collinear type II SPDC in a standard twin-photon polarization interferometer, previously used in numerous experiments ${ }^{30}$ and shown in Fig. 2. The twin photons, with the state shown in Eq. (2), impinge on the input port of a nonpolarizing beam splitter, so that on $50 \%$ of the trials the two photons are separated into the two output ports of the beam splitter. ${ }^{33}$ In the remainder of the trials, the two photons emerge together from the beam splitter out of one of the ports, but such cases do not contribute to coincidence measurements and thus may be ignored. Photons emerging from the one of the output ports of the beam splitter are directed to the sample under test and are then directed to polarization analyzer $A_{1}$ followed by single-photon detector $D_{1}$. Photons emerging from the other output port are directed to polarization analyzer $\mathrm{A}_{2}$ followed by single-photon detector $\mathrm{D}_{2}$. A coincidence circuit registers the coincidence rate $N_{c}$ of the detectors $\mathrm{D}_{1}$ and $\mathrm{D}_{2}$, which is proportional to the fourth-order coherence function of the fields at the detectors. $^{34,35}$ In this subsection, we demonstrate how this unentangled twin-photon polarization interferometer yields ellipsometric measurements.

We first introduce a matrix formalism that facilitates the derivation of the fields at the detectors. We begin by defining a twin-photon Jones vector that represents the field operators of the signal and idler in two spatially distinct modes. If $\hat{a}_{s}(\omega)$ and $\hat{a}_{i}\left(\omega^{\prime}\right)$ are the boson annihilation operators for the signal-frequency mode $\omega$ and idlerfrequency mode $\omega^{\prime}$, respectively, then the twin-photon Jones vector of the field following the beam splitter is

$$
\hat{\mathbf{J}}_{1}=\left(\begin{array}{c}
j\left\{-\hat{\mathbf{A}}_{s}(\omega)+\hat{\mathbf{A}}_{i}\left(\omega^{\prime}\right)\right\} \\
\hat{\mathbf{A}}_{s}(\omega)+\hat{\mathbf{A}}_{i}\left(\omega^{\prime}\right)
\end{array}\right),
$$

where $\hat{\mathbf{A}}_{s}(\omega)=\hat{a}_{s}(\omega)\left(\begin{array}{l}1 \\ 0\end{array}\right)$ and $\hat{\mathbf{A}}_{i}\left(\omega^{\prime}\right)=\hat{a}_{i}\left(\omega^{\prime}\right)\left(\begin{array}{l}0 \\ 1\end{array}\right){ }^{36}$ The vectors $\left(\begin{array}{l}1 \\ 0\end{array}\right)$ (horizontal) and $\left(\begin{array}{l}0 \\ 1\end{array}\right)$ (vertical) are the familiar Jones vectors representing orthogonal polarization states. $^{37}$ The operators $\hat{\mathbf{A}}_{s}(\omega)$ and $\hat{\mathbf{A}}_{i}\left(\omega^{\prime}\right)$ thus are annihilation operators that include the vectorial polarization

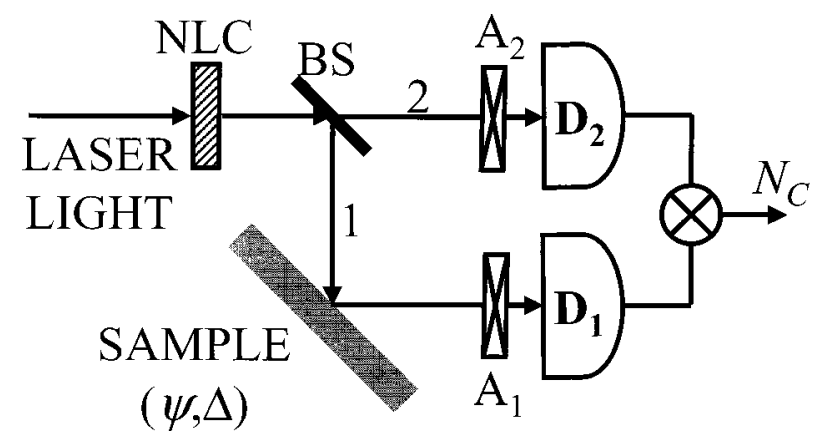

Fig. 2. Unentangled twin-photon ellipsometer: NLC stands for nonlinear crystal; $\mathrm{BS}$ is a nonpolarizing beam splitter; $\mathrm{A}_{1}$ and $\mathrm{A}_{2}$ are linear polarization analyzers; $\mathrm{D}_{1}$ and $\mathrm{D}_{2}$ are single-photon detectors; and $N_{c}$ is the coincidence rate. information of the field mode. The first element in $\hat{\mathbf{J}}_{1}$, $j\left\{-\hat{\mathbf{A}}_{s}(\omega)+\hat{\mathbf{A}}_{i}\left(\omega^{\prime}\right)\right\}$, represents the annihilation operator of the field in beam 1 , which is a superposition of signal and idler field operators. The second element in $\hat{\mathbf{J}}_{1}$, $\hat{\mathbf{A}}_{s}(\omega)+\hat{\mathbf{A}}_{i}\left(\omega^{\prime}\right)$, is the annihilation operator of the field in beam 2 .

We now define a twin-photon Jones matrix that represents the action of linear deterministic optical elements, placed in the two beams, on the polarization of the field as follows:

$$
\mathbf{T}=\left[\begin{array}{ll}
\mathbf{T}_{11} & \mathbf{T}_{12} \\
\mathbf{T}_{21} & \mathbf{T}_{22}
\end{array}\right]
$$

where $\mathbf{T}_{k l}(k, l=1,2)$ is the familiar $2 \times 2$ Jones matrix that represents the polarization transformation performed by a linear deterministic optical element. The indices refer to the spatial modes of the input and output beams. For example, $\mathbf{T}_{11}$ is the Jones matrix of an optical element placed in beam 1 whose output is also in beam 1 , whereas $\mathbf{T}_{21}$ is the Jones matrix of an optical element placed in beam 1 whose output is in beam 2 , and similarly for $\mathbf{T}_{12}$ and $\mathbf{T}_{22}$. In most cases, when an optical element is placed in beam 1 and another in beam $2, \mathbf{T}_{12}=\mathbf{T}_{21}$ $=$ 0. An exception is, e.g., a beam splitter with beams 1 and 2 incident on its two input ports, or other optical components that mix the spatial modes of the two beams. The twin-photon Jones matrix $\mathbf{T}$ transforms a twinphoton Jones vector $\hat{\mathbf{J}}_{1}$ into $\hat{\mathbf{J}}_{2}$ according to $\hat{\boldsymbol{J}}_{2}=\mathbf{T} \hat{\mathbf{J}}_{1}$.

Applying this formalism to the arrangement in Fig. 2, assuming that beams 1 and 2 impinge on the two polarization analyzers $A_{1}$ and $A_{2}$ directly (in absence of the sample), the twin-photon Jones matrix is given by

$$
\mathbf{T}_{p}=\left[\begin{array}{cc}
\mathbf{P}\left(-\theta_{1}\right) & \mathbf{0} \\
\mathbf{0} & \mathbf{P}\left(\theta_{2}\right)
\end{array}\right],
$$

where

$$
\mathbf{P}(\theta)=\left[\begin{array}{cc}
\cos ^{2} \theta & \cos \theta \sin \theta \\
\cos \theta \sin \theta & \sin ^{2} \theta
\end{array}\right],
$$

and $\theta_{1}$ and $\theta_{2}$ are the angles of the axes of the analyzers with respect to the horizontal direction. In this case the twin-photon Jones vector following the analyzers is therefore

$$
\begin{aligned}
\hat{\mathbf{J}}_{2} & =\mathbf{T}_{p} \hat{\mathbf{J}}_{1}=\left(\begin{array}{c}
j \mathbf{P}\left(-\theta_{1}\right)\left\{-\hat{\mathbf{A}}_{s}(\omega)+\hat{\mathbf{A}}_{i}\left(\omega^{\prime}\right)\right\} \\
\mathbf{P}\left(\theta_{2}\right)\left\{\hat{\mathbf{A}}_{s}(\omega)+\hat{\mathbf{A}}_{i}\left(\omega^{\prime}\right)\right\}
\end{array}\right) \\
& =\left(\begin{array}{c}
j\left\{-\cos \theta_{1} \hat{a}_{s}(\omega)+\sin \theta_{1} \hat{a}_{i}\left(\omega^{\prime}\right)\right\}\left(\begin{array}{c}
\cos \theta_{1} \\
-\sin \theta_{1}
\end{array}\right) \\
\left\{\cos \theta_{2} \hat{a}_{s}(\omega)+\sin \theta_{2} \hat{a}_{i}\left(\omega^{\prime}\right)\right\}\left(\begin{array}{c}
\cos \theta_{2} \\
\sin \theta_{2}
\end{array}\right)
\end{array}\right) .
\end{aligned}
$$

Using the twin-photon Jones vector $\hat{\mathbf{J}}_{2}$, one can obtain expressions for the fields at the detectors. The positivefrequency components of the field at detectors $D_{1}$ and $D_{2}$, denoted $\hat{\mathbf{E}}_{1}^{+}$and $\hat{\mathbf{E}}_{2}^{+}$, respectively, are given by 


$$
\begin{aligned}
\hat{\mathbf{E}}_{1}^{+}(t)= & j\left\{-\cos \theta_{1} \int d \omega \exp (-j \omega t) \hat{a}_{s}(\omega)\right. \\
& \left.+\sin \theta_{1} \int d \omega^{\prime} \exp \left(-j \omega^{\prime} t\right) \hat{a}_{i}\left(\omega^{\prime}\right)\right\}\left(\begin{array}{c}
\cos \theta_{1} \\
-\sin \theta_{1}
\end{array}\right), \\
\hat{\mathbf{E}}_{2}^{+}(t)= & \left\{\cos \theta_{2} \int d \omega \exp (-j \omega t) \hat{a}_{s}(\omega)\right. \\
& \left.+\sin \theta_{2} \int d \omega^{\prime} \exp \left(-j \omega^{\prime} t\right) \hat{a}_{i}\left(\omega^{\prime}\right)\right\}\left(\begin{array}{c}
\cos \theta_{2} \\
\sin \theta_{2}
\end{array}\right),
\end{aligned}
$$

while the negative frequency components are given by their Hermitian conjugates. With these fields one can show that the coincidence rate $N_{c} \propto \sin ^{2}\left(\theta_{1}-\theta_{2}\right)$ using the expressions developed in Appendix A.

Consider now that the sample, assumed to have frequency-independent reflection coefficients, is placed in the optical arrangement illustrated in Fig. 2, and that the polarizations of the downconverted photons are along the eigenpolarizations of the sample. The effect of the sample, placed in beam 1 , may be represented by the following twin-photon Jones matrix:

$$
\mathbf{T}_{s}=\left[\begin{array}{cc}
\mathbf{R} & \mathbf{0} \\
\mathbf{0} & \mathbf{I}
\end{array}\right],
$$

where

$$
\mathbf{R}=\left[\begin{array}{cc}
\tilde{r}_{1} & 0 \\
0 & \widetilde{r}_{2}
\end{array}\right]
$$

(the justification for using this matrix to represent the action of the sample is provided in Appendix A), $\mathbf{I}$ is the 2 $\times 2$ identity matrix, and $\widetilde{r}_{1}$ and $\widetilde{r}_{2}$ are the complex reflection coefficients of the sample described earlier. The twin-photon Jones vector after reflection from the sample and passage through the polarization analyzers is given by

$$
\begin{aligned}
\hat{\mathbf{J}}_{3} & =\mathbf{T}_{p} \mathbf{T}_{s} \hat{\mathbf{J}}_{1} \\
= & \left(\begin{array}{c}
j\left\{-\widetilde{r}_{1} \cos \theta_{1} \hat{a}_{s}(\omega)+\widetilde{r}_{2} \sin \theta_{1} \hat{a}_{i}\left(\omega^{\prime}\right)\right\}\left(\begin{array}{c}
\cos \theta_{1} \\
-\sin \theta_{1}
\end{array}\right) \\
\left\{\cos \theta_{2} \hat{a}_{s}(\omega)+\sin \theta_{2} \hat{a}_{i}\left(\omega^{\prime}\right)\right\}\left(\begin{array}{c}
\cos \theta_{2} \\
\sin \theta_{2}
\end{array}\right)
\end{array}\right),
\end{aligned}
$$

which results in

$$
\begin{aligned}
\hat{\mathbf{E}}_{1}^{+}(t)= & j\left\{-\widetilde{r}_{1} \cos \theta_{1} \int d \omega \exp (-j \omega t) \hat{a}_{s}(\omega)\right. \\
& \left.+\widetilde{r}_{2} \sin \theta_{1} \int d \omega^{\prime} \exp \left(-j \omega^{\prime} t\right) \hat{a}_{i}\left(\omega^{\prime}\right)\right\}\left(\begin{array}{c}
\cos \theta_{1} \\
-\sin \theta_{1}
\end{array}\right),
\end{aligned}
$$

with $\hat{\mathbf{E}}_{2}^{+}(t)$ identical to Eq. (8), since there is no sample in this beam.

Finally, using the expressions developed in Appendix A, it is straightforward to show that

$$
\begin{aligned}
N_{c}= & C\left[\tan ^{2} \psi \cos ^{2} \theta_{1} \sin ^{2} \theta_{2}+\sin ^{2} \theta_{1} \cos ^{2} \theta_{2}\right. \\
& \left.-2 \tan \psi \cos \Delta \cos \theta_{1} \cos \theta_{2} \sin \theta_{1} \sin \theta_{2}\right],
\end{aligned}
$$

where the constant of proportionality $C$ depends on the efficiencies of the detectors and the duration of accumulation of coincidences. One can obtain $C, \psi$, and $\Delta$ with a minimum of three measurements with different analyzer settings, e.g., $\theta_{2}=0^{\circ}, \theta_{2}=90^{\circ}$, and $\theta_{2}=45^{\circ}$, while $\theta_{1}$ remains fixed at any angle except $0^{\circ}$ and $90^{\circ}$.

If the sample is replaced by a perfect mirror, the coincidence rate in Eq. (13) becomes a sinusoidal pattern of $100 \%$ visibility, $C \sin ^{2}\left(\theta_{1}-\theta_{2}\right)$, as previously indicated. In practice, by judicious control of the apertures placed in the downconverted beams, visibilities close to $100 \%$ can be obtained.

To understand the need for temporal compensation discussed previously, we rederive Eq. (13), which assumes full compensation, when a birefringent compensator is placed in one of the arms of the configuration:

$$
\begin{aligned}
N_{c}=C & {\left[\tan ^{2} \psi \cos ^{2} \theta_{1} \sin ^{2} \theta_{2}+\sin ^{2} \theta_{1} \cos ^{2} \theta_{2}\right.} \\
& -2 \tan \psi \cos \Delta \cos \theta_{1} \cos \theta_{2} \sin \theta_{1} \\
& \left.\times \sin \theta_{2} \Phi(\tau) \cos \left(\omega_{0} \tau\right)\right] .
\end{aligned}
$$

Here $\tau$ is the birefringent delay, $\omega_{0}$ is half the pump frequency, and $\Phi(\tau)$ is the Fourier transform of the SPDC normalized power spectrum. When $\tau=0$, we recover Eq. (13), whereas when $\tau$ is larger than the inverse of the SPDC bandwidth, the third term that includes $\Delta$ becomes zero, and thus $\Delta$ cannot be determined.

The drawback of the arrangement illustrated in Fig. 2 is the requirement for a beam splitter, as in classical interferometric ellipsometry. Any deviation from the assumed symmetric reflectance/transmittance of this device will impair the measurements and necessitate the use of a reference sample for calibration.

\section{B. Entangled Twin-Photon Ellipsometer}

As in classical interferometry, the configuration in the previous subsection uses a beam splitter as a means of creating the multiple paths that lead to interference. We now show that one can construct an interferometer that makes use of quantum entanglement, which then dispenses with the beam splitter. This has the salutary effect of keeping $100 \%$ of the incoming photon flux (rather than 50\%) while eliminating the requirement of characterizing it. Moreover, no other optical elements are introduced, so one need not be concerned with the characterization of any components. This is a remarkable feature of entanglement-based quantum interferometry.

The NLC is adjusted to produce SPDC in a type II noncollinear configuration, as illustrated in Fig. 3. Following the procedure discussed in the previous subsection, it is straightforward to show that the resulting coincidence rate is given by

$$
\begin{aligned}
N_{c}= & C\left[\tan ^{2} \psi \cos ^{2} \theta_{1} \sin ^{2} \theta_{2}+\sin ^{2} \theta_{1} \cos ^{2} \theta_{2}\right. \\
& \left.+2 \tan \psi \cos \Delta \cos \theta_{1} \cos \theta_{2} \sin \theta_{1} \sin \theta_{2}\right] .
\end{aligned}
$$

This expression is virtually identical to the one presented in Eq. (13) (except for the substitution of the plus sign for the minus sign in the last term). An interesting feature 


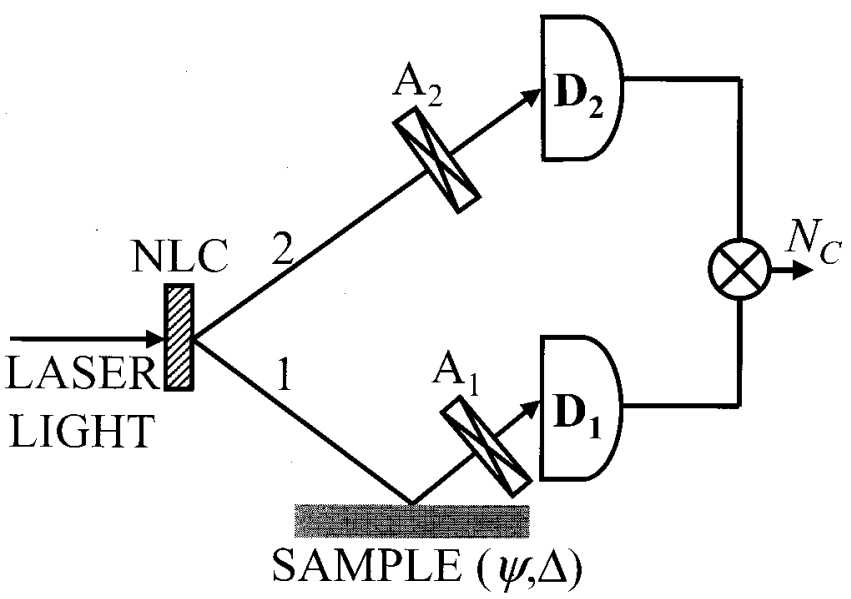

Fig. 3. Entangled twin-photon ellipsometer.

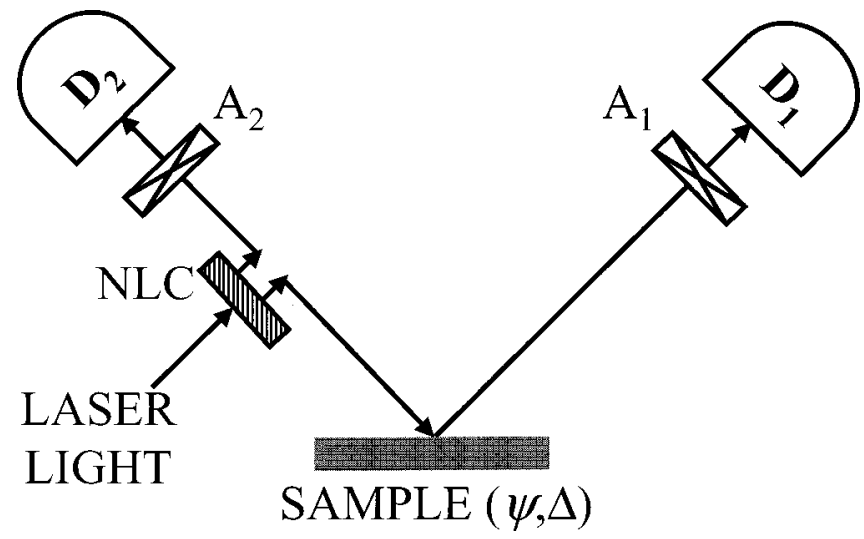

Fig. 4. Unfolded version of the entangled twin-photon ellipsometer displayed in Fig. 3.

of this interferometer is that it is not sensitive to an overall mismatch in the length of the two arms of the setup, and this increases the robustness of the arrangement.

An illuminating way of representing the action of the entangled twin-photon quantum ellipsometer is readily achieved by redrawing Fig. 3 in the unfolded configuration shown in Fig. 4. Using the advanced-wave interpretation, which was suggested by Klyshko in the context of twin-photon imaging, ${ }^{38}$ the coincidence rate for photons at $\mathrm{D}_{1}$ and $\mathrm{D}_{2}$ may be obtained by tracing light waves originating from $\mathrm{D}_{2}$ to the NLC and then onto $\mathrm{D}_{1}$ upon reflection from the sample. With this interpretation, the configuration in Fig. 4 becomes geometrically similar to the classical ellipsometer. Although none of the optical components usually associated with interferometers (beam splitters and wave plates) are present in this scheme, interferometry is still effected through the entanglement of the source.

An advantage of this setup over its idealized null ellipsometric counterpart, discussed in Section 2, is that the two arms of the ellipsometer are separate, and the light beams traverse them independently in different directions. This allows various instrumentation errors of the classical setup to be circumvented. For example, placing optical elements before the sample causes beam deviation errors $^{39}$ when the faces of the optical components are not exactly parallel. This leads to an error in the angle of incidence and, consequently, errors in the estimated parameters. In our case no optical components are placed between the source (NLC) and the sample; any desired polarization manipulation may be performed in the other arm of the entangled twin-photon ellipsometer. Furthermore, one can change the angle of incidence to the sample easily and repeatedly.

A significant drawback of classical ellipsometry is the difficulty of fully controlling the polarization of the incoming light. A linear polarizer is usually employed at the input of the ellipsometer, but the finite extinction coefficient of this polarizer causes errors in the estimated parameters. $^{2}$ In the entangled twin-photon ellipsometer the polarization of the incoming light is dictated by the phase-matching conditions of the nonlinear interaction in the NLC. The polarizations defined by the orientation of the optical axis of the NLC play the role of the input polarization in classical ellipsometry. The NLC is aligned for type II SPDC so that only one polarization component of the pump generates SPDC, whereas the orthogonal (undesired) component of the pump does not (since it does not satisfy the phase-matching conditions). The advantage is therefore that the downconversion process ensures the stability of polarization along a particular direction.

\section{CONCLUSION}

Classical ellipsometric measurements are limited in their accuracy by virtue of the need for an absolutely calibrated source and detector. Mitigating this limitation requires the use of a well-characterized reference sample in a null configuration.

Twin-photon ellipsometry, which makes use of simultaneously emitted photon pairs, is superior because it removes the need for a reference sample. Nevertheless, the unentangled twin-photon ellipsometer requires that the optical components employed in the interferometric arrangement be well characterized.

We have demonstrated that entangled twin-photon ellipsometry is self-referencing and therefore eliminates the necessity of constructing an interferometer altogether. The underlying physics that leads to this remarkable result is the presence of fourth-order (coincidence) quantum interference of the photon pairs in conjunction with nonlocal polarization entanglement.

Our proposed entangled twin-photon ellipsometer is subject to the same shot-noise-limited, as well as angularly resolved, precision that is obtained with traditional ellipsometers (interferometric and null systems, respectively), but removes the limitation in accuracy that results from the necessity of using a reference sample in traditional ellipsometers.

Since the SPDC source is inherently broadband, narrow-band spectral filters must be used to ensure that the ellipsometric data are measured at a specific frequency. Spectroscopic data can be obtained by employing a bank of such filters. Alternatively, techniques from Fourier-transform spectroscopy may be used to directly make use of the broadband nature of the source in ellipsometric measurements. 


\section{APPENDIX A}

We investigate the effect that reflection from a sample has on the quantized-field operators. We model the sample as a lossless beam splitter, with complex reflection coefficient $\widetilde{r}$ and complex transmission coefficient $\tilde{t}$, that transforms the input field operators $\hat{a}_{1}$ and $\hat{a}_{v}$ into output field operators $\hat{b}_{1}$ and $\hat{b}_{v}$ according to

$$
\hat{b}_{1}=j \widetilde{r} \hat{a}_{1}+\tilde{t} \hat{a}_{v}, \quad \hat{b}_{v}=\tilde{t} \hat{a}_{1}+j \widetilde{r} \hat{a}_{v},
$$

where $|\widetilde{t}|^{2}+|\widetilde{r}|^{2}=1$ (so that the bosonic commutation relations are preserved for $\hat{b}_{1}$ and $\hat{b}_{v}$ ), $\hat{a}_{1}$ is the annihilation operator of a single mode of the incident optical field, and $\hat{a}_{v}$ is the annihilation operator of the vacuum entering the other port of the beam splitter.

The coincidence rate at the detectors at times $t_{1}$ and $t_{2}$ is given by

$$
G\left(t_{1}, t_{2}\right)=\left\langle\Psi\left|\hat{E}_{1}{ }^{(-)}\left(t_{1}\right) \hat{E}_{2}{ }^{(-)}\left(t_{2}\right) \hat{E}_{2}{ }^{(+)}\left(t_{2}\right) \hat{E}_{1}{ }^{(+)}\left(t_{1}\right)\right| \Psi\right\rangle,
$$

where $\quad \hat{E}_{1}{ }^{(+)}(t)=\int d \omega \exp (-j \omega t) \hat{b}_{1}(\omega), \quad \hat{E}_{2}{ }^{(+)}(t)$ $=\int d \omega \exp (-j \omega t) \hat{a}_{2}(\omega)$, and $|\Psi\rangle$ is the twin-photon state at the output of the NLC:

$$
|\Psi\rangle=\int d \omega \varphi\left(\omega, \omega_{p}-\omega\right)\left|1_{\omega}, 1_{\omega_{p}-\omega}, 0_{\omega}\right\rangle .
$$

The first element in the ket corresponds to the signal mode at frequency $\omega$, the second element corresponds to the idler mode at frequency $\omega_{p}-\omega$ (conservation of energy ensures that the signal and idler frequencies add up to the pump frequency $\omega_{p}$ ), and the third element corresponds to the vacuum mode at frequency $\omega$ at the other input port of the beam splitter that represents the sample. The function $\varphi\left(\omega, \omega_{p}-\omega\right)$ is the probability amplitude of the possible combinations of frequencies for pairs of signal and idler modes emitted by the NLC.

Inserting the identity operator

$$
\Sigma_{n_{1}, n_{2}, n_{3}}\left|n_{1}, n_{2}, n_{3}\right\rangle\left\langle n_{1}, n_{2}, n_{3}\right|
$$

(represented in the Fock basis of the Hilbert space spanned by the signal, idler, and vacuum fields) into Eq. (A2) gives

$$
\begin{aligned}
G\left(t_{1}, t_{2}\right)= & \sum_{n_{1}, n_{2}, n_{3}}\left\langle\Psi\left|\hat{E}_{1}{ }^{(-)}\left(t_{1}\right) \hat{E}_{2}{ }^{(-)}\left(t_{2}\right)\right| n_{1}, n_{2}, n_{3}\right\rangle \\
& \times\left\langle n_{1}, n_{2}, n_{3}\left|\hat{E}_{2}^{(+)}\left(t_{2}\right) \hat{E}_{1}^{(+)}\left(t_{1}\right)\right| \Psi\right\rangle \\
= & \sum_{n_{1}, n_{2}, n_{3}}\left|\left\langle n_{1}, n_{2}, n_{3}\left|\hat{E}_{2}{ }^{(+)}\left(t_{2}\right) \hat{E}_{1}^{(+)}\left(t_{1}\right)\right| \Psi\right\rangle\right|^{2} .
\end{aligned}
$$

It is straightforward to show that using the state in Eq. (A3) results in all terms in the summation in Eq. (A4) vanishing except for the term where $n_{1}=n_{2}=n_{3}=0$, so that $G\left(t_{1}, t_{2}\right)=\left|\left\langle 0,0,0\left|\hat{E}_{2}{ }^{(+)}\left(t_{2}\right) \hat{E}_{1}{ }^{(+)}\left(t_{1}\right)\right| \Psi\right\rangle\right|^{2}$, and also results in the terms containing $\tilde{t} \hat{a}_{v}$ vanishing. Thus in coincidence measurements, the effect of reflection from the sample appears as a direct multiplication of the relevant operators by the suitable complex reflection coefficient, which justifies the use of the matrix in Eq. (10) when two orthogonal polarizations of the fields (and thus two corresponding complex reflection coefficients) are taken into consideration. Note that the detectors actually record a time-averaged coincidence rate $N_{c}$ since the response time for optical detectors is usually much longer than the inverse bandwidth of the function $\varphi\left(\omega, \omega_{p}\right.$ - $\omega$ ) (see Ref. 35 for details).

\section{ACKNOWLEDGMENTS}

This work was supported by the National Science Foundation (NSF) and by the Center for Subsurface Sensing and Imaging Systems (CenSSIS), an NSF engineering research center.

A. V. Sergienko's e-mail address is alexserg@bu.edu.

\section{REFERENCES}

1. P. Drude, "Bestimmung optischer Konstanten der Metalle," Ann. Physik Chemie 39, 481-554 (1890).

2. R. M. A. Azzam and N. M. Bashara, Ellipsometry and Polarized Light (North-Holland, Amsterdam, 1977).

3. H. G. Tompkins and W. A. McGahan, Spectroscopic Ellipsometry and Reflectometry (Wiley, New York, 1999).

4. A. Rothen, "The ellipsometer, an apparatus to measure thicknesses of thin surface films," Rev. Sci. Instrum. 16, 26-30 (1945).

5. A. B. Winterbottom, "Optical methods of studying films on reflecting bases depending on polarisation and interference phenomena," Trans. Faraday Soc. 42, 487-495 (1946).

6. M. Mansuripur, "Ellipsometry," Opt. Photon. News 11(4), 52-56 (2000)

7. D. N. Klyshko, "Coherent decay of photons in a nonlinear medium," Pis'ma Zh. Eksp. Teor. Fiz. 6, 490-492 (1967) [Sov. Phys. JETP Lett. 6, 23-25 (1967)].

8. S. E. Harris, M. K. Oshman, and R. L. Byer, "Observation of tunable optical parametric fluorescence," Phys. Rev. Lett. 18, 732-735 (1967).

9. T. G. Giallorenzi and C. L. Tang, "Quantum theory of spontaneous parametric scattering of intense light," Phys. Rev. 166, 225-233 (1968).

10. D. A. Kleinman, "Theory of optical parametric noise," Phys. Rev. 174, 1027-1041 (1968).

11. D. N. Klyshko, Photons and Nonlinear Optics (Gordon and Breach, New York, 1988).

12. A. Zeilinger, "Experiment and the foundations of quantum physics," Rev. Mod. Phys. 71, S288-S297 (1999).

13. E. S. Fry and T. Walther, "Fundamental tests of quantum mechanics," in Advances in Atomic, Molecular, and Optical Physics, B. Bederson and H. Walther, eds. (Academic, Boston, 2000), Vol. 42, pp. 1-27.

14. D. N. Klyshko, "Utilization of vacuum fluctuations as an optical brightness standard," Kvant. Elektron. (Moscow) 4, 1056-1062 (1977) [Sov. J. Quantum Electron. 7, 591-595 (1977)].

15. A. Migdall, R. Datla, A. V. Sergienko, and Y. H. Shih, "Absolute detector quantum efficiency measurements using correlated photons," Metrologia 32, 479-483 (1995).

16. D. Branning, A. L. Migdall, and A. V. Sergienko, "Simultaneous measurement of group and phase delay between two photons," Phys. Rev. A 62, 063808 (2000).

17. A. F. Abouraddy, K. C. Toussaint, Jr., A. V. Sergienko, B. E. Saleh, and M. C. Teich, "Ellipsometric measurements by use of photon pairs generated by spontaneous parametric downconversion," Opt. Lett. 26, 1717-1719 (2001).

18. A. K. Ekert, J. G. Rarity, P. R. Tapster, and G. M. Palma, "Practical quantum cryptography based on two-photon interferometry," Phys. Rev. Lett. 69, 1293-1295 (1992).

19. A. V. Sergienko, M. Atatüre, Z. Walton, G. Jaeger, B. E. A. Saleh, and M. C. Teich, "Quantum cryptography using femtosecond-pulsed parametric down-conversion," Phys. Rev. A 60, R2622-R2625 (1999).

20. T. Jennewein, C. Simon, G. Weihs, H. Weinfurter, and A. 
Zeilinger, "Quantum cryptography with entangled photons," Phys. Rev. Lett. 84, 4729-4732 (2000).

21. D. Bouwmeester, J.-W. Pan, K. Mattle, M. Eibl, H. Weinfurter, and A. Zeilinger, "Experimental quantum teleportation," Nature 390, 575-579 (1997).

22. D. Boschi, S. Branca, F. De Martini, L. Hardy, and S. Popescu, "Experimental realization of teleporting an unknown pure quantum state via dual classical and EinsteinPodolsky-Rosen channels," Phys. Rev. Lett. 80, 1121-1125 (1998).

23. B. E. A. Saleh, S. Popescu, and M. C. Teich, "Generalized entangled-photon imaging," in Proceedings of the Ninth Annual Meeting of the IEEE Lasers and Electro-Optics Society, P. Zory, ed. (IEEE, Piscataway, N.J., 1996), Vol. 1, pp. 362363.

24. M. B. Nasr, A. F. Abouraddy, M. C. Booth, B. E. A. Saleh, A. V. Sergienko, M. C. Teich, M. Kempe, and R. Wollenschensky, "Biphoton focusing for two-photon excitation," Phys. Rev. A 65, 023816 (2002).

25. A. F. Abouraddy, B. E. A. Saleh, A. V. Sergienko, and M. C. Teich, "Entangled-photon Fourier optics," J. Opt. Soc. Am. B (to be published).

26. M. C. Teich and B. E. A. Saleh, "Observation of sub-Poisson Franck-Hertz light at 253.7 nm,” J. Opt. Soc. Am. B 2, 275282 (1985).

27. M. C. Teich and B. E. A. Saleh, "Photon bunching and antibunching," Prog. Opt. 26, 1-104 (1988).

28. M. C. Teich and B. E. A. Saleh, "Squeezed and antibunched light," Phys. Today 43(6), 26-34 (1990).

29. P. G. Kwiat, K. Mattle, H. Weinfurter, A. Zeilinger, A. V. Sergienko, and Y. Shih, "New high-intensity source of polarization-entangled photon pairs," Phys. Rev. Lett. 75, 4337-4341 (1995).
30. A. V. Sergienko, Y. H. Shih, and M. H. Rubin, "Experimental evaluation of a two-photon wave packet in type-II parametric downconversion," J. Opt. Soc. Am. B 12, 859-862 (1995).

31. U. Fano, "Description of states in quantum mechanics by density matrix and operator techniques," Rev. Mod. Phys. 29, 74-93 (1957).

32. A. F. Abouraddy, B. E. A. Saleh, A. V. Sergienko, and M. C. Teich, "Degree of entanglement for two qubits," Phys. Rev. A 64, 050101(R) (2001).

33. R. A. Campos, B. E. A. Saleh, and M. C. Teich, "Quantummechanical lossless beam splitter: $\mathrm{SU}(2)$ symmetry and photon statistics," Phys. Rev. A 40, 1371-1384 (1989).

34. R. J. Glauber, "The quantum theory of optical coherence," Phys. Rev. 130, 2529-2539 (1963).

35. B. E. A. Saleh, A. F. Abouraddy, A. V. Sergienko, and M. C. Teich, "Duality between partial coherence and partial entanglement," Phys. Rev. A 62, 043816 (2000).

36. R. J. Glauber, "Optical coherence and photon statistics," in Quantum Optics and Electronics, Les Houches, C. DeWitt A. Blandin, and C. Cohen-Tannoudji, eds. (Gordon and Breach, New York, 1965).

37. B. E. A. Saleh and M. C. Teich, Fundamentals of Photonics (Wiley, New York, 1991).

38. D. N. Klyshko, "A simple method of preparing pure states of an optical field, of implementing the Einstein-PodolskyRosen experiment, and of demonstrating the complementarity principle," Usp. Fiz. Nauk 154, 133-152 (1988) [Sov. Phys. Usp. 31(1), 74-85 (1988)].

39. J. R. Zeidler, R. B. Kohles, and N. M. Bashara, "Beam deviation errors in ellipsometric measurements; an analysis" Appl. Opt. 13, 1938-1945 (1974). 